\title{
PROCESS AND SYSTEMS Do medical admission pro formas improve the completeness of documentation? A multisite observational study
}

\author{
Authors: Nicholas Smallwood, ${ }^{A}$ Joe Russell, ${ }^{B}$ Rachel Forbes-Pyman ${ }^{C}$ and Anna Coates ${ }^{D}$
}

There is little evidence as to whether clerking pro formas are more or less effective than continuation sheets for the acute medical admission clerking process. This two site audit aimed to assess the effect of introducing clerking pro formas on the completeness of documentation in 32 areas. Data were collected at two sites both pre- and post-introduction of two different clerking pro formas, and a two-tailed z-test used to assess the significance in changes in documentation at each individual site and combined across both sites. Site one showed improvements in $23 / 32$ areas, with $7 / 32$ (21.9\%) being significant; three areas saw a decline and $1 / 32$ showed a statistically significant decline. Site two showed improvement across $25 / 32$ sites with 19 (59.4\%) being significant; there were no areas showing less complete documentation. Cross-site analysis showed improvements in 30/32 categories, with 15 (46.9\%) being significant; one area showed a non-significant decline. The greatest percentage improvement was seen in documentation of cardiopulmonary resuscitation / escalation decisions - an important consideration in this clinically unstable population. The introduction of clerking pro formas across two sites improved the completeness of documentation in a number of areas. This data supports the widespread introduction of clerking pro formas as a tool to improve the completeness of admission documentation.

KEYWORDS: Pro forma, clerking pro forma, medical audit, admissions, documentation

\section{Introduction}

Despite their widespread use within many hospital admission units, there is minimal published evidence on the utility of preprinted pro formas to alter the quality of documentation during

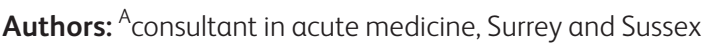
Healthcare NHS Trust, East Surrey Hospital, Redhill, UK; ${ }^{B}$ registrar in acute medicine, Surrey and Sussex Healthcare NHS Trust, East Surrey Hospital, Redhill, UK; C physician associate in acute medicine, Surrey and Sussex Healthcare NHS Trust, East Surrey Hospital, Redhill, UK; ${ }^{\mathrm{D}} \mathrm{CT} 2$ anaesthetics, University College London Hospitals NHS Foundation Trust, London, UK the adult medicine clerking process. The authors found one clinica letter relating to a single centre study looking at the completeness of documentation before and after introduction of a preprinted pro forma, which suggested improvements in a number of areas when the pro forma was used. ${ }^{1}$ No other data relating to adult medical admissions were found in the published literature.

Traditionally the admission clerking would have been completed on blank history sheets but these are increasingly being replaced by dedicated pro formas with defined subheadings and structures. The Royal College of Physicians (RCP) has recommended that all admission documentation be recorded using a standardised pro forma ${ }^{2}$ and in 2013 they published guidance on the standard headings that should be utilised as part of the admission process. ${ }^{3}$ However, they left it to individual organisations as to which headings should be used in which scenario, acknowledging a one-size-fits-all approach would not work. There is evidence that improving the structure of medical records improves doctors' performance, ${ }^{4}$ but this may not necessarily translate to a more complete medical record being created.

In this study, we attempted to determine if the introduction of a preprinted clerking pro forma altered the completeness (and so perhaps the quality) of admission documentation. Two separate sites were audited before and after introduction of a pro forma, to determine if it had a positive or negative effect on documentation of 32 pieces of clinical data. Each site was using blank history sheets initially and subsequently introduced preprinted pro formas. The pro formas introduced were different across each site and so some of the results suggest a generic 'pro forma effect' rather than results specific to the document itself, which is beneficial when considering the generalisability of this data.

\section{Methods}

The same audit process was conducted across two sites at two separate times. A random selection of medical notes was accessed across general medical wards on a given day to ensure a crosssection of days of admission, admitting clinicians and admitting consultants. The case notes were reviewed up to the point of post-take ward round and the presence or absence of data in 32 separate areas were recorded (see Table 1 for categories). These areas of documentation were chosen by the authors as representing best practice and encompass many of the headings highlighted in the RCP document. ${ }^{2}$ 
Table 1. 32 categories used as best practice for documentation

\begin{tabular}{ll} 
Demographics & X-ray results \\
Age & ABG/VBG results \\
Presenting complaint & AMTS \\
History of presenting complaint & CPR/escalation plan \\
Past medical history & Senior review \\
Drug history & Initial diagnosis \\
Where/who drug history elicited from & Diagnosis following PTWR \\
Allergies & Observations \\
Family history & Respiratory exam \\
Social history & Cardiology exam \\
Smoking/alcohol status & Abdominal exam \\
Exercise tolerance & Neurological exam \\
Impression/problem list & Date (of clerking) \\
Blood results & Time (of clerking) \\
Urinalysis results & Doctors name \\
ECG results & Consultant \\
\hline
\end{tabular}

$\mathrm{ABG}=$ arterial blood gas; $\mathrm{AMTS}=$ abbreviated mental test score; $\mathrm{CPR}=$ cardiopulmonary resuscitation; $E C G$ = electrocardiogram; $P T W R=$ post-take ward round; $\mathrm{VBG}=$ venous blood gas
Across both sites a second audit of random inpatient notes was undertaken after introduction of a preprinted clerking pro forma, to compare the completeness of documentation. The data were analysed both within sites to assess individual changes with each pro forma and across both sites to look for changes that were more applicable to pro formas in general (a generic 'pro forma effect'). The z-test was used for statistical analysis (two-tailed, $95 \%$ confidence interval, $p<0.05$ ).

Clinicians within each hospital (except the supervising audit lead) were not informed that the audit was taking place to minimise the chance of bias.

This work involved clinical audits and as such ethical approval was not required and consent for participation was not sought.

\section{Results}

In site one, a total of 50 sets of notes were analysed both pre- and post-pro forma, while in site two a total of 75 sets of notes were examined. Data were available for $100 \%$ (4000) data points.

Across both sites individually there was a tendency to improvement in completeness of documentation with introduction of the pro forma, with only one area across both sites showing a statistically significant decline. These data are summarised in Table 2.

\section{Combined site analysis}

Complete data points are available for 125 sets of notes both pre and post introduction of medical pro formas across the two sites. The overall documentation rate improved from $63.4 \%$ (2534/4000) before the pro formas were introduced to $73.5 \%$ (2940/4000) afterwards; an absolute improvement of $10.1 \%$ and

Table 2. Changes in completeness of documentation by site for 32 categories

\begin{tabular}{|c|c|c|c|c|c|}
\hline & Site 1 & Site 2 & & Site 1 & Site 2 \\
\hline Demographics & Decline $^{a}$ & Improve $^{a}$ & $X$-ray results & Neutral & Improve \\
\hline Age & Improve & Improve $^{a}$ & ABG/VBG results & Improve & Improve \\
\hline Presenting complaint & Improve & Improve $^{a}$ & AMTS & Improve $^{a}$ & Decline \\
\hline History of presenting complaint & Neutral & Improve & CPR/escalation plan & Improve $^{a}$ & Decline \\
\hline Past medical history & Improve & Improve $^{a}$ & Senior review & Improve & Improve $^{a}$ \\
\hline Drug history & Improve & Improve & Initial diagnosis & Improve $^{a}$ & Improve \\
\hline Where/who drug history elicited from & Improve & Decline & Diagnosis following PTWR & Improve & Improve $^{a}$ \\
\hline Allergies & Improve & Improve $^{a}$ & Observations & Improve $^{a}$ & Improve $^{a}$ \\
\hline Family history & Improve & Improve $^{a}$ & Respiratory exam & Neutral & Improve $^{a}$ \\
\hline Social history & Improve & Improve & Cardiology exam & Improve & Improve $^{a}$ \\
\hline Smoking/alcohol status & Improve & Improve $^{a}$ & Abdominal exam & Improve & Improve $^{a}$ \\
\hline Exercise tolerance & Decline & Improve $^{a}$ & Neurological exam & Neutral & Improve $^{a}$ \\
\hline Impression/problem list & Improve & Improve & Date (of clerking) & Improve $^{a}$ & Decline \\
\hline Blood results & Neutral & Decline & Time (of clerking) & Neutral & Decline \\
\hline Urinalysis results & Decline & Improve $^{a}$ & Doctors name & Improve $^{a}$ & Improve $^{a}$ \\
\hline ECG results & Improve & Improve $^{a}$ & Consultant & Improve $^{a}$ & Decline \\
\hline
\end{tabular}


a relative improvement of $15.9 \%$. This improvement is statistically significant $(p<0.001)$.

When looking at individual categories, improvements in completeness of documentation were seen in 30/32 (93.8\%), with $15 / 32(46.9 \%)$ showing statistical significance. One category (3.1\%) showed no change and one $(3.1 \%)$ showed a decline in completeness of documentation. This latter result was not statistically significant.

The largest improvements in percentage terms were seen with the documentation of cardiopulmonary resuscitation (CPR) / escalation plans (190\% improvement), family history (78.9\%) and smoking/alcohol history (61\%). The only category to see a decline in completeness of documentation was time of clerking $(3.5 \%$ decline). These data are summarised in Table 3.

\section{Discussion}

There has previously been very little published data on the utility of clerking pro formas on the quality of documentation among adult medical admissions, despite their widespread use and the $\mathrm{RCP}$ recommendation to use them as a standard part of the admission process. The data presented here show that pro formas

Table 3. Combined results across both sites for change in completeness of documentation with absolute numbers (out of 125), relative percentage changes and a number needed to treat analysis

\begin{tabular}{|c|c|c|c|c|c|}
\hline & Pre & Post & Change expressed as NNT & Relative change & p-value \\
\hline Demographics & 108 & 108 & - & Neutral & 1 \\
\hline Age & 100 & 119 & 6.58 & $19 \%$ improve & $<0.001$ \\
\hline Presenting complaint & 112 & 125 & 9.62 & $11.6 \%$ improve & $<0.001$ \\
\hline History of presenting complaint & 123 & 125 & 62.5 & $1.6 \%$ improve & 0.15 \\
\hline Past medical history & 106 & 123 & 7.35 & $16.0 \%$ improve & $<0.001$ \\
\hline Drug history & 109 & 117 & 15.63 & $7.3 \%$ improve & 0.08 \\
\hline Where/who drug history elicited from & 15 & 17 & 62.5 & $13.3 \%$ improve & 0.7 \\
\hline Allergies & 84 & 111 & 4.63 & $32.1 \%$ improve & $<0.001$ \\
\hline Family history & 33 & 59 & 4.81 & $78.8 \%$ improve & $<0.001$ \\
\hline Social history & 106 & 114 & 15.63 & $7.5 \%$ improve & 0.11 \\
\hline Smoking/alcohol status & 62 & 100 & 3.29 & $61.3 \%$ improve & $<0.001$ \\
\hline Exercise tolerance & 37 & 43 & 20.83 & $16.2 \%$ improve & 0.41 \\
\hline Impression/problem list & 111 & 117 & 20.83 & $5.4 \%$ improve & 0.18 \\
\hline Blood results & 110 & 112 & 62.5 & $1.8 \%$ improve & 0.68 \\
\hline Urinalysis results & 40 & 46 & 20.83 & $15 \%$ improve & 0.42 \\
\hline ECG results & 73 & 100 & 4.63 & $37.0 \%$ improve & $<0.001$ \\
\hline X-ray results & 75 & 86 & 11.36 & $14.7 \%$ improve & 0.14 \\
\hline ABG/VBG results & 43 & 57 & 8.93 & $32.6 \%$ improve & 0.07 \\
\hline AMTS & 33 & 45 & 10.42 & $36.4 \%$ improve & 0.1 \\
\hline CPR/escalation plan & 10 & 29 & 6.58 & $190 \%$ improve & 0.001 \\
\hline Senior review & 43 & 54 & 11.36 & $25.6 \%$ improve & 0.15 \\
\hline Initial diagnosis & 39 & 47 & 15.63 & $20.5 \%$ improve & 0.28 \\
\hline Diagnosis following PTWR & 88 & 108 & 6.25 & $22.7 \%$ improve & 0.002 \\
\hline Observations & 101 & 117 & 7.81 & $15.8 \%$ improve & 0.002 \\
\hline Respiratory exam & 115 & 125 & 12.5 & $8.7 \%$ improve & 0.001 \\
\hline Abdominal exam & 113 & 124 & 11.36 & $9.7 \%$ improve & 0.001 \\
\hline Neurological exam & 63 & 78 & 8.33 & $23.8 \%$ improve & 0.05 \\
\hline Date (of clerking) & 120 & 123 & 41.67 & $2.5 \%$ improve & 0.25 \\
\hline Time (of clerking) & 114 & 110 & -31.25 & $3.5 \%$ decline & 0.4 \\
\hline Doctor's name & 106 & 125 & 6.58 & $17.9 \%$ improve & $<0.001$ \\
\hline Consultant & 33 & 52 & 6.58 & $57.6 \%$ improve & 0.01 \\
\hline
\end{tabular}


improve the completeness of documentation compared to blank history sheets, which may also suggest the quality of those admission documents is improved. Particularly significant improvements were seen in the documentation of CPR/escalation plans, smoking and family history, while when combining both sites there were no areas of documentation which showed a significant decline in completion rate following introduction of the pro formas.

Similar data has been published previously in a number of other hospital specialties. Preprinted pro formas have increased completeness of documentation in paediatric admissions, ${ }^{5}$ asthma admissions, ${ }^{6}$ stroke admissions, ${ }^{7}$ psychiatry admissions ${ }^{8}$ and surgical admissions ${ }^{9}$. In addition, pro formas have been shown to improve the diagnosis of patients admitted with a hand injury, ${ }^{10}$ and improve compliance with the Best Practice Tariff among hip fracture patients. ${ }^{11}$ All of these reports are single-site observational studies; this is the first evidence to demonstrate improvements across two sites with two separate pro formas, which helps reduce the bias associated with the individual design of the pro forma (which varies across almost all hospital sites). No previous data were found relating to changes in completeness of documentation across more than one site. The cross-site analysis is important as it suggests some of the improvements may be a generic "pro forma effect' rather than down to the individual design of the pro formas which may significantly impact on the completeness of documentation. It might be imagined that a poorly designed pro forma would hinder some documentation, while a well-designed form should encourage it. The combination of data across two individual pro formas strengthens the generalisability of this data. It also suggests that many of the improvements are down to the use of a pro forma per se, rather than just a well-designed single pro forma in a single unit as many of the previous data could be partly attributed to.

Perhaps the most important improvement in documentation was around CPR and escalation decisions. Although the overall numbers of documented decisions remained relatively low (29/125 from 10/125 records), it was a large percentage increase. This patient group (acute medical admissions) are by definition medically unstable and increasingly frail. Early decision making around ceilings of care and appropriateness of cardiopulmonary resuscitation can only be of benefit. Other notable improvements not previously mentioned were seen in the documentation of allergy status ( $32 \%$ improvement) and of the diagnosis following the post-take ward round ( $22 \%$ improvement). The former clearly has important connotations for patient safety. It is disappointing that the completion rate was not $100 \%$ for both of these categories, but an improvement with pro formas is clearly welcome. The only area across either site which showed a significant decline in completeness in documentation was for demographics in site one (23.3\% decline). It is not possible to explain this finding, but is reassuring to note that combining the data for both sites saw no change in this variable (perhaps implying a local pro forma design issue).

Another area where pro formas have potential benefits over freehand documentation is where safety checklists are recommended to ensure that important decisions are made (for example, venous thromboembolism prophylaxis, dementia/ delirium assessments and antibiotic stewardship). As we have shown in this study, it is likely that including specific prompts on pro formas about these categories will improve their documentation (although they are areas that were not specifically assessed here). The use of ward round checklists is recommended by the RCP ${ }^{13}$ and including them as part of a preprinted pro forma for the post take ward round and subsequent reviews should ensure that they are utilised.

It is not possible to say categorically that because the completeness of documentation was improved by the introduction of pro formas, the quality (or safety) was also improved - although it seems intuitive. Increasing amounts of information will allow the clinician to form a more holistic picture of the patient and so make a more appropriate clinical decision. Perhaps more importantly, it will allow a more complete written record to form part of the handover process when patient care is transferred from one team to another, which happens with increasing regularity in modern medicine. Finally, it has been hypothesised that improving the completeness of documentation may lead to a reduction in adverse events, ${ }^{14}$ which could only be beneficial from a patient safety viewpoint.

Without qualitatively assessing the quality of clerkings before and after introductions of pro formas, it is not possible to rule out the possibility that some areas may be limited by their introduction. For example, the ability to completely document a neurological exam may be limited by a poorly designed pro forma, or the completeness of a past medical history may be restricted if only a few common condition check boxes are provided. A qualitative assessment was not undertaken with this study and indeed would have only been of use for each individual institution rather than generalisable across the UK. It is therefore imperative that each trust which introduces a pro forma (or has one already) regularly assesses the quality of clerkings undertaken with their pro formas and undertakes to make improvements in them through a plan-do-study-act (PDSA) approach.

There are clearly methodological issues with this type of data. Observational data are not free from bias and the relatively small numbers available for analysis make the statistical significance less robust. Importantly, it is not possible with this work to state categorically that the quality of admission clerkings increased or that patient outcomes improved with the pro forma, although there are surrogate markers that the former may be true. An improvement in patient outcomes would be hard to show without a large-scale randomised controlled trial, which is both impractical and unlikely to be undertaken. It would be helpful to be able to show whether users of the pro forma felt that there were improvements with their introduction, although a large survey of more than 1000 clinicians published by the RCP stated clinicians were strongly in favour of structured pro formas over free-hand clerking during the admissions process. ${ }^{2}$

\section{Conclusions}

Overall these data provide clear reassurance that the introduction of pro formas improves the completeness of documentation of the admission clerking and probably also the quality of those clerkings with a particular benefit seen around the documentation of 'Do not attempt cardiopulmonary resuscitation' and escalation decisions. This is particularly welcome given that the RCP has suggested their mandatory introduction within medicine and there previously being very little published evidence of their effect on the clerking process. 


\section{References}

1 O'Driscoll R, Al-Nuaimi D. Medical admission records can be improved by the use of a structured proforma. Clin Med 2003;3:385-6.

2 Carpenter I, Ram MB, Croft GP, Williams JG. Medical records and record-keeping standards. Clin Med 2007;7:328-31.

3 Health and Social Care Information Centre, Academy of Medical Royal Colleges. Standards for the clinical structure and content of patient records. London: HSCIC, 2013.

4 Mann R, Williams ]. Standards in medical record keeping. Clin Med 2003;3:329-32.

5 Goodyear HM, Lloyd BW. Can admission notes be improved by using preprinted assessment sheets? Qual Health Care 1995:4:190-3.

6 Robinson SM, Harrison BDW, Lambert MA. Effect of a preprinted form on the management of acute asthma in an accident and emergency department. J Accid Emerg Med 1996:13:93-7.

7 Patel S. Improving documentation within the acute stroke unit: Introducing a stroke specific clerking proforma. BMJ Qual Improv Rep 2015;4:u208852.w3847.

8 Wethers G, Brown J. Does an admission booklet improve patient safety? J Ment Health 2011:20:438-44.
9 Ehsanullah J, Ahmad U, Solanki K, Healy J, Kadoglou N. The surgical admissions proforma: Does it make a difference? Ann Med Surg 2015:4:53-7.

10 Ding BT, Rajaratnam V. The utility of using a hand clerking sheet for initial assessment of hand-related injuries. J Orthop Surg (Hong Kong) 2017:25:1-5.

11 Chamberlain M, Pugh $\mathrm{H}$. Improving inpatient care with the introduction of a hip fracture pathway. BMJ Qual Imp Rep 2015;4: u204075.w2786.

12 Lyons JM, Martinez JA, O'Leary JP. Medical malpractice matters: medical record M \& Ms. J Surg Educ 2009;66:113-7.

13 Royal College of Physicians, Royal College of Nursing. Ward rounds in medicine: principles for best practice. London: RCP, 2012.

14 Zegers M, de Bruijne MC, Spreeuwenberg P et al. Quality of patient record keeping: an indicator of the quality of care? BMJ Qual Saf 2011:20:314-8.

Address for correspondence: Dr Nicholas Smallwood, Department of Acute Medicine, Surrey and Sussex Healthcare NHS Trust, East Surrey Hospital, Canada Avenue, Redhill RH1 5RH, UK.

Email: nicholas.smallwood@nhs.net

\section{Royal College} of Physicians

\section{Physicians and war}

War has been a frequent feature of British life over the 500 years of the Royal College of Physicians' existence, and has an important place in the histories of both the RCP and medicine.

Order by phone or online

Tel +44 (0)20 30751358

or visit www.rcplondon.ac.uk/shop

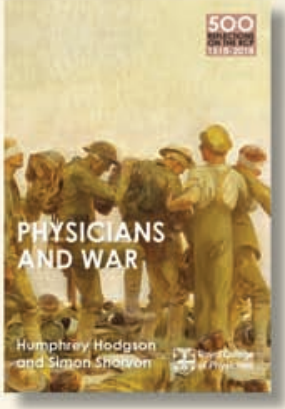

Price: $£ 12$

(Postage and packaging not included)

$10 \%$ discount for fellows and members.

$15 \%$ discount for foundation doctors and students 